\title{
Glutamine deficiency shifts the asthmatic state toward neutrophilic airway inflammation
}

\author{
June-Mo Kim ${ }^{1}$, Yoo-Na Im $^{1}$, Yun-Jo Chung ${ }^{2}$, Jung-ho Youm ${ }^{1}$, Suhn-Young Im $^{3}$, Myung \\ Kwan $\mathrm{Han}^{1}$, and Hern Ku Lee ${ }^{1}$ \\ ${ }^{1}$ Jeonbuk National University Medical School \\ ${ }^{2}$ Jeonbuk National University \\ ${ }^{3}$ Chonnam National University
}

July 21,2021

\begin{abstract}
Background: The administration of L-glutamine (Gln) suppresses allergic airway inflammation via the rapid upregulation of MAPK phosphatase (MKP)-1, which functions as a negative regulator of inflammation by deactivating p38 and JNK mitogenactivated protein kinases (MAPKs). However, the role of endogenous Gln remains to be elucidated. Therefore, we investigated the mechanism by which endogenous Gln regulates MKP-1 induction and allergic airway inflammation in an ovalbumin-based murine asthma model. Methods: We depleted endogenous Gln levels using l- $\gamma$-glutamyl- $p$-nitroanilide (GPNA), an inhibitor of the Gln transporter ASCT2, and glutamine synthetase small interfering (si)RNA. Lentivirus expressing MKP-1 was injected to achieve overexpression of MKP-1. Asthmatic phenotypes were assessed using our previously developed ovalbumin-based murine model, which is suitable for examining sequential asthmatic events, including neutrophil infiltration. Gln levels were analyzed using a Gln assay kit. Results: GPNA or glutamine synthetase siRNA successfully depleted endogenous Gln levels. Importantly, homeostatic MKP-1 induction did not occur at all, which resulted in prolonged p38 MAPK and cytosolic phospholipase A 2 (cPLA 2 ) phosphorylation in Gln-deficient mice. Gln deficiency augmented all examined asthmatic reactions, but it exhibited a strong bias toward increasing the neutrophil count, which was not observed in MKP-1-overexpressing lungs. This neutrophilia was inhibited by a cPLA 2 inhibitor and a leukotriene B4 inhibitor, but not by dexamethasone. Conclusion: Gln deficiency leads to the impairment of MKP-1 induction and activation of p38 MAPK and cPLA 2 , resulting in the augmentation of neutrophilic, more so than eosinophilic, airway inflammation.
\end{abstract}

\section{Hosted file}

Gln defi_manuscript x-unmarked.docx available at https://authorea.com/users/426899/articles/ 531244-glutamine-deficiency-shifts-the-asthmatic-state-toward-neutrophilic-airwayinflammation

\section{Hosted file}

Gln_defi_Figure x Unmarked.pptx available at https://authorea.com/users/426899/articles/ 531244-glutamine-deficiency-shifts-the-asthmatic-state-toward-neutrophilic-airwayinflammation 